O45 (continued)

Evaluation: ODPHP conducted usability testing on the toolkit messages and is collecting comments at conferences, as was requested in the audience research.

Conclusions and Implications: This toolkit supports health professionals as important partners in sharing Guidelines recommendations with patients. Health professionals can initiate discussions, provide referrals to registered dietitians and nutrition educators, and encourage patients to follow-up in seeking nutrition counseling.

Funding: US Department of Health and Human Services

\section{Are We Listening? How to Engage Low- Income Caregivers with Nutrition Messaging} ChristopherSneed,PhD, csneed@utk.edu, The University of Tennessee Extension, 1801 Downtown West Boulevard, Knoxville, TN 37919; Karen Franck, PhD, The University of Tennessee Extension; Michelle Vineyard, $P h D, L D N, R D$

Objective: To identify preferred nutrition information sources, messages, and communication channels for limited-resource caregivers of school-aged children.

Study Design, Setting, Participants, Intervention: Participants were recruited from telephone surveys that screened for eligibility based on being caregivers of children ages preschool to fifth grade and residing in a household receiving federal assistance. Nine focus groups were conducted in community locations.

Outcome Measures and Analysis: Shannon and Weaver's Mathematical Theory of Communication framed questions that focused on concepts related to trusted sources for nutrition information, messages most likely to foster healthy eating, and preferred communication channels. Focus group recordings were transcribed and analyzed for common themes by two researchers. Discrepancies were discussed and resolved.

Results: 108 individuals participated. They identified trusted sources of nutrition information that included professionals such as nutritionists, doctors, and teachers as well as health-focused celebrities. Participants were most interested in information regarding meal preparation, benefits of healthy eating, and simple strategies for making healthy choices. They preferred internet and social media and posters placed in agency lobbies.

Conclusions and Implications: Low-income participants trusted information from nutritionists but prefer to receive nutrition information through social media and online sources rather than the traditional face-to-face methods. Practical information - recipes and money saving strategies - were most desired. Findings provide essential insight into strategies for communicating nutrition content to limited-resource caregivers. This presentation will focus on how nutrition education professionals can use these findings to tailor education to engage the target population using sources, messaging and communication channels most preferred by the group.

Funding: Supplemental Nutrition Assistance Program Education

\section{Don't Reinvent the Wheel: Ohio SNAP- Ed's Social Marketing Campaign as a Model for Health Promotion to Low-Income Families}

ElizabethHustead, MS, hustead.7@osu.edu, The Ohio State University, 1787 Neil Avenue, Columbus, OH 43210; Kara Golis, $B A, R D$, The Ohio State University; Brian Butler, MPH; Joyce Counihan, MA, CPS Baobab; Anna Claudia Zubieta, PhD, The Ohio State University

Objective: To thoroughly document the process of the SNAP-Ed social marketing campaign in order to generate a social marketing model that could be replicated by SNAP-Ed or other social service programs across the country.

Target Audience: Any agency or group interested in launching a social marketing campaign to promote health and nutrition.

Theory, Prior Research, Rationale: Social marketing is a popular and effective tool for achieving behavioral changes in a population. Health and nutrition-related campaigns include Oregon State University's FoodHero and the Iowa Nutrition Network's Pick a Better Snack.

Description: Ohio SNAP-Ed is partnering with the State Nutrition Action Committee (SNAC) to design a social marketing campaign to promote fruit and vegetable consumption among low-income (at or below $185 \%$ of federal poverty) families with children. The entire process will be carefully documented and translated into a model for other agencies and groups to apply to their own health promotion issues.

Evaluation: The efficacy of the campaign will be determined by the target audience's knowledge and recognition of the campaign following the statewide rollout. In addition, behavior change in the form of increased fruit and vegetable consumption will be measured. These success metrics will be used to optimize the model.

Conclusions and Implications: While the results of many social marketing campaigns are well documented and show that social marketing is extremely effective, the complete process has not ever been documented in such a way as to make a campaign easily reproducible. By using the SNAP-Ed campaign to generate a model, this research will help define the future of social marketing for health promotion.

Funding: Supplemental Nutrition Assistance Program Education

\section{Dietary Guidelines for Americans: The Past, Present, and Future}

Kellie O. Casavale, PhD, RD, kellie.casavale@hhs.org, U.S. Department of Health and Human Services, 1101

Wootton Parkway, Rockville, MD 20852;

Eve Essery Stoody, PhD, U.S. Department of Agriculture; Elizabeth Rahavi, BS, RD; Frances Bevington, MA, U.S.

Department of Health and Human Services; Collette Rihane, RD, MS, U.S. Department of Agriculture; Richard D. Olson, MPH, MD, U.S. Department of Health and Human Services 